\title{
Limited Angle C-Arm Tomography and Segmentation for Guidance of Atrial Fibrillation Ablation Procedures
}

\author{
Dirk Schäfer ${ }^{1}$, Carsten Meyer ${ }^{1}$, Roland Bullens ${ }^{2}$, \\ Axel Saalbach ${ }^{1}$, and Peter Eshuis ${ }^{2}$ \\ 1 Philips Research, Röntgenstraße 24-26, 22335 Hamburg, Germany \\ 2 Philips Healthcare, Veenpluis 6, 5684 PC Best, The Netherlands \\ dirk.schaefer@philips.com
}

\begin{abstract}
Angiographic projections of the left atrium (LA) and the pulmonary veins (PV) acquired with a rotational C-arm system are used for 3D image reconstruction and subsequent automatic segmentation of the LA and PV to be used as roadmap in fluoroscopy guided LA ablation procedures. Acquisition of projections at high oblique angulations may be problematic due to increased collision danger of the detector with the right shoulder of the patient. We investigate the accuracy of image reconstruction and model based roadmap segmentation using limited angle C-arm tomography. The reduction of the angular range from $200^{\circ}$ to $150^{\circ}$ leads only to a moderate increase of the segmentation error from $1.5 \mathrm{~mm}$ to $2.0 \mathrm{~mm}$ if matched conditions are used in the segmentation, i.e. the model based segmentation is trained on images reconstructed with the same angular range as the test images. The minor decrease in accuracy may be outweighed by clinical workflow improvement, gained when large C-arm angulations can be avoided.
\end{abstract}

Keywords: Atrial fibrillation, ablation guidance, 3D roadmap.

\section{Introduction}

Electric isolation of the pulmonary veins (PV) by ablation is a standard procedure for treatment of atrial fibrillation (AF). Patient specific 3D surface models of the left atrium (LA) are used as roadmap overlay to support the fluoroscopy guided intervention. The roadmap can either be integrated from a segmentation of a pre-interventional CT or MR scan, or it can be generated directly on the C-arm system with a 3D Atriography scan (3D-ATG) [1]. A 3D-ATG acquisition consists of a rotational acquisition covering an angular range of 200 degree from $100^{\circ} \mathrm{RAO}$ (right anterior oblique) to $100^{\circ} \mathrm{LAO}$ (left anterior oblique). The left atrium and the pulmonary veins (LAPV) are filled with contrast agent and are positioned in the iso-center of the $\mathrm{C}$-arm system. The acquired angiograms are used for 3D image reconstruction which is followed by automatic model based segmentation. 
The patient is shifted asymmetrically to the right with respect to the C-arm system in order to position the LA in the iso-center. In this setup, the acquisition of the projections between $\approx 60^{\circ} \mathrm{RAO}$ and $100^{\circ} \mathrm{RAO}$ may be problematic due to increased collision danger of the detector with the patient shoulder.

Hence, reconstructions from a limited tomographic angle are desired. However, filtered back-projection (FBP) reconstructions from limited angles suffer from decreased sharpness and image blur along the direction perpendicular to the missing projection directions. This problem has been adressed using iterative reconstruction techniques with edge preserving regularization [2] or minimization of the total variation norm [3]. In the case of C-arm imaging additional problems arise due to the limited detector size and the lateral truncation of the projections. The incomplete projection data can be handeled in iterative algorithms by reconstructing very large image sizes [4], which is depreciated for interventional use due to time constraints. Furthermore iterative methods are known to be more sensitive to inconsistencies due to motion or varying contrast agent filling.

Therefore, we keep the FBP method and optimize the segmentation method with respect to the given clinical task. By focussing on the clinical needs Bachar et al. 5] showed e.g. for interventional head and neck C-arm imaging sufficient image quality in terms of the localization accuracy. They obtained a localization error of several features representing different contrast levels below $2.5 \mathrm{~mm}$ for tomographic angles $\geq 90^{\circ}$ using standard FBP. In this work, we concentrate on the clinical need of delineation of the LA and PV combined with improved workflow of limited angle tomography. The issue of limited angle tomography is adressed by matched learning of the segmentation algorithm using standard FBP reconstruction. We investigate the accuracy of 3D-ATG image reconstruction and subsequent model based segmentation of the LAPV using rotational acquisitions with reduced angular ranges to minimize the collision danger and hence improve the clinical workflow. In particular, we present a detailed quantitative evaluation of the segmentation performance in matched and mismatched training and test conditions, acquired with different angular ranges.

\section{Methods}

\subsection{D Rotational Angiography}

All intra-procedural imaging was performed using a flat-detector C-arm system (Allura Xper FD10, Philips Medical Systems, Inc., Best, The Netherlands). The contrast agent (CA) is either administered by a right-sided or a left sided injection. A right sided injection is performed by administration of CA into the right atrium (RA) or pulmonary artery (PA) with a delay of the X-ray scan of $3-5$ seconds by manual-triggering accounting for the time needed for the CA to arrive in the left atrium. A left sided injection is performed directly into the left atrium together with hyper-pacing or administration of adenosine to disable the pumping of the heart and maintain the CA inside the LA. During injection, patients were instructed to hold their breath at end-expiration until completion 
of the rotational run. The rotational X-ray data sets are acquired with 116 projections on $\approx 200^{\circ}$ from $100^{\circ} \mathrm{RAO}$ to $100^{\circ} \mathrm{LAO}$ over 4 seconds at a sampling rate of 30 frames/s. For our investigation, we reduce the angular range of the acquisition by skipping some projections at the endings of the source trajectory.

\subsection{Image Reconstruction}

The flat detector is fixed at maximum distance from the $\mathrm{X}$-ray source with a fan angle of $9^{\circ}$ and rotated in propeller mode around the patient along a calibrated circular arc, which is parameterized by the path length $\lambda \in \Lambda$. The angular range $\Delta$ of the source path $\Lambda$ covers $200^{\circ}$ from $100^{\circ} \mathrm{RAO}$ to $100^{\circ} \mathrm{LAO}$ in the full acquisition and is symmetrically reduced to four different settings $\Delta=170^{\circ}, 150^{\circ}, 130^{\circ}, 110^{\circ}$. The distance between source $\mathbf{S}$ and the detector center $\mathbf{D}$ is given by $D$. The normalized vector $\hat{\mathbf{d}}(\lambda)$ points from $\mathbf{D}(\lambda)$ to the source. The detector $v$-axis is parallel to the rotational axis and the $u$-axis is parallel to the trajectory tangent vector. The cone beam projection data of the object $f_{0}$ is denoted by $\mathcal{X}(u, v, \lambda)$ :

$$
\mathcal{X}(u, v, \lambda)=\int_{0}^{\infty} f_{0}(\mathbf{S}(\lambda)+l \hat{\mathbf{e}}(u, v, \lambda)) \mathrm{d} l,
$$

where $\hat{\mathbf{e}}(u, v, \lambda)$ is the unit vector from the source position $\mathbf{S}(\lambda)$ to the detector element $\mathbf{E}(u, v, \lambda)$. The corresponding length is denoted by $\overline{S E}$. The projection data is extended prior to filtering using the Lewitt-method to mitigate truncation artifacts. The $3 \mathrm{D}$ image $f$ is reconstructed using cone-beam filtered back-projection [6]:

$$
f(\mathbf{x})=\int_{\Lambda} \frac{D}{|(\mathbf{x}-\mathbf{S}) \cdot \hat{\mathbf{d}}|^{2}} \int_{-\infty}^{\infty} w\left(\lambda, u^{\prime}\right) \frac{D}{\overline{S E}(\mathbf{x})} \mathcal{X}\left(u^{\prime}, v, \lambda\right) h_{R}\left(u-u^{\prime}\right) \mathrm{d} u^{\prime} \mathrm{d} \lambda,
$$

where $h_{R}$ is the ramp filter and $u, v$ are the detector coordinates of the forward projected point $\mathbf{x}$. The weighting function $w(\lambda, u)$ is given by the short scan Parker weights in case of $\Delta=200^{\circ}$. If $\Delta$ is reduced, $w$ is given by $w=\frac{\pi}{\int_{\Lambda(\Delta)} \mathrm{d} \lambda}$ to correct for the decreasing brightness in the reconstructed image when using less projections.

\subsection{Model Based Segmentation}

We use a model-based segmentation framework developed for segmenting the left atrium and pulmonary veins [1]. Mesh adaptation is performed after initial automatic localization by iterating two steps until the mesh reaches a steady state 7]. First, boundaries are detected. Then, the mesh is deformed by minimizing the weighted sum of an external energy $E_{\text {ext }}$ and an internal energy $E_{\text {int }}$. The external energy pulls the mesh to the detected boundaries. The internal energy penalizes deformations which deviate from a reference shape (e.g., mean shape) undergoing a geometric transformation (e.g., rigid or affine) accounting for pose and shape variability. 
Image Intensity Calibration: To compensate for image intensity variations across images in 3D-ATG data, we compute the image histogram and determine the low and high $2 \%$ percentiles. The intensity values within the remaining histogram are then linearly re-scaled to a suitable reference interval, e.g., [0, 1].

The LAPV + trachea model: We use a deforming mesh consisting of an LAPV part and a trachea part shown in Fig. 2. The trachea has been added to facilitate carina-based registration of the $3 \mathrm{D}$ model overlay to the live fluoroscopic projections during intervention [8]. The LAPV part represents the endocardial surface of the LA and the surface of the four main PV trunks. This mesh part is made of 1330 vertices which form 2717 triangles. The trachea part consists of 1076 nodes forming 2148 triangles. With this mesh topology, a reference (ground truth) mesh was obtained semi-automatically using a bootstrapping approach, i.e. a sequence of intermediate models was created based on a subset of the available volumes. Using these models, segmentations were obtained for each of the 57 3D-ATG data sets and then refined by a clinical expert by manual adaptation to match the observable image boundaries as closely as possible.

Multi-stage segmentation: A multi-stage adaptation framework is applied to increase robustness. The first step is the initialization of the barycenter of the model based on a generalized Hough transform (GHT) [9]. In a second step, the mesh is deformed parametrically, by applying individual similarity transformations for the LAPV and the trachea mesh parts to minimize the external energy $E_{\text {ext }}$. This compensates for pose misalignment as well as for variations in the relative orientation of the LAPV and the trachea between patients. Finally, a deformable adaptation step is performed as described above (including $E_{\text {int }}$ ) to account for more local variations of the patients anatomy.

Optimized Boundary Detection: Since vertex correspondence is preserved during mesh adaptation, we can assign a locally optimal boundary detection function to each mesh triangle using the Simulated Search approach 10. Specifically, the magnitude of the image gradient (projected onto the triangle normal vector) together with several additional constraints on the gray values and image characteristics across the boundary are used for boundary detection. Suitable candidates for the constraints (e.g. gray values left or right of the boundary) and reasonable acceptance intervals for the constraints are estimated from the training images. The simulated search selects the optimal boundary detection function for each triangle from all candidates.

Assessment of Segmentation Accuracy and Mesh Comparison: The segmentation accuracy is assessed by measuring the Euclidean surface-to-patch distance $\epsilon_{i, n}$ between the automatic segmentation of the 3D-ATG data and the corresponding reference mesh. $\epsilon_{i, n}$ is defined as the distance between triangle center $i$ of the adapted 3D-ATG mesh for patient $n$ to an anatomically corresponding patch of maximum geodesic radius $r=10 \mathrm{~mm}$ of the reference mesh and vice versa [7]. Averaging over triangles $i$ gives the error $\epsilon_{n}$ per patient, and further averaging 
over the 57 patients gives the mean error $\epsilon_{\text {mean }}$. The triangle positions near the artificial cut-planes which define the distal bounds of the truncated pulmonary veins and those of the trachea are excluded from the error measurement [1].

\section{Results}

In this study, 57 rotational ATG data sets have been used, which were acquired at three different clinical sites in Europe and the USA according to the protocol described in Section 2.1 Each data set has been reconstructed by restricting the projections to the 5 different angular ranges $\Delta=200^{\circ}, 170^{\circ}, 150^{\circ}, 130^{\circ}, 110^{\circ}$. For each angular range $\Delta$, a separate model for automatic segmentation has been generated, i.e. optimal boundary detection features and a GHT (see Sec. 2.3) have been trained on the corresponding reconstructed images. For training and evaluation of the model, 10-fold cross-validation has been used. In addition, each trained model has been evaluated on the reconstructions of the corresponding test patients with the other angular ranges, to assess the effect of a mismatch between the angular range used in training and testing. A summary of the mean segmentation error $\epsilon_{\text {mean }}$ with respect to the reference meshes (see Sec. 2.3) for the various scenarios is given in Table 1 and visualized in Fig. 1.

Table 1. Average segmentation error $\epsilon_{\text {mean }}$ of the whole mesh in $[\mathrm{mm}]$ for various training and test angular ranges

\begin{tabular}{c|ccccc} 
& \multicolumn{5}{|c}{ Test on } \\
Training on & $200^{\circ}$ & $170^{\circ}$ & $150^{\circ}$ & $130^{\circ}$ & $110^{\circ}$ \\
\hline $200^{\circ}$ & $\mathbf{1 . 5 1}$ & 1.61 & 2.53 & 4.35 & 5.82 \\
$170^{\circ}$ & $\mathbf{1 . 4 8}$ & $\mathbf{1 . 5 9}$ & 2.41 & 3.86 & 5.30 \\
$150^{\circ}$ & 1.59 & 1.66 & $\mathbf{1 . 9 8}$ & 3.33 & 4.86 \\
$130^{\circ}$ & 2.11 & 1.86 & 2.05 & $\mathbf{3 . 0 0}$ & 3.58 \\
$110^{\circ}$ & 1.98 & 1.92 & 2.24 & $\mathbf{2 . 9 4}$ & $\mathbf{3 . 2 2}$
\end{tabular}

For each angular range used for reconstructing the test image, the optimal training model (leading to the minimal test error) is highlighted by bold numbers in Table 1. For three angular ranges $\left(\Delta=170^{\circ}, 150^{\circ}, 110^{\circ}\right)$, this minimal error is obtained for matched conditions, i.e., a model trained on the same angular range as used in the test. For the remaining two ranges the difference to the minimal error is very small (bold and italic numbers). In the case of train and test with $200^{\circ}$, the segmentation error of $1.5 \mathrm{~mm}$ is similar to other published results 1$] 12$.

The following conclusions can be drawn: First, a reduction of the angular range from $200^{\circ}$ to $170^{\circ}$ has — if at all — only a minimal influence on the segmentation performance (from $1.5 \mathrm{~mm}$ to $1.6 \mathrm{~mm}$ ). Second, if smaller angular ranges (< $\left.170^{\circ}\right)$ are used in the test images, segmentation performance decreases significantly (Figure 1). Better segmentation performance can be obtained by using a segmentation model trained in matched conditions, i.e., on images reconstructed 
with the same angular range as the test image. Thereby, for $150^{\circ}$ reconstructions, a segmentation error of about $2 \mathrm{~mm}$ is obtained. Third, these findings hold for the whole LAPV plus trachea mesh, but also for the individual anatomical structures of the mesh, as shown in Table 2. Fourth, if the reconstruction angle is further reduced to $130^{\circ}$ or $110^{\circ}$, a segmentation error of about $3 \mathrm{~mm}$ is obtained in matched conditions. Models trained on low reconstruction angles are generally more robust in mismatched test conditions, while not being optimal for large reconstruction angles.

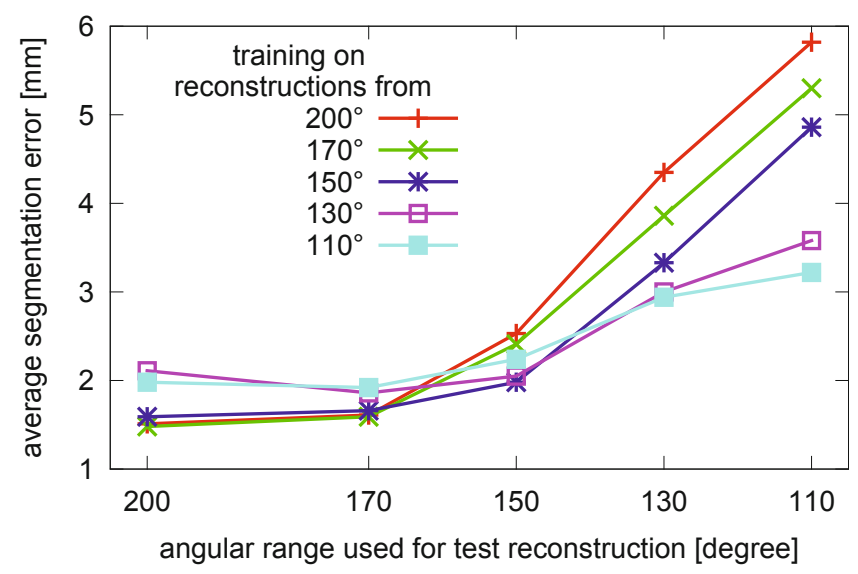

Fig. 1. Average segmentation error $\epsilon_{\text {mean }}$ of the whole mesh for various training and test angular ranges

Table 2. Average segmentation error of the individual mesh parts in $[\mathrm{mm}]$ for various training and test angular ranges

\begin{tabular}{l|ccc|ccc|ccc|} 
& \multicolumn{3}{|c|}{ Test on $200^{\circ}$} & \multicolumn{3}{c|}{ Test on $170^{\circ}$} & \multicolumn{3}{c|}{ Test on $150^{\circ}$} \\
& Train: & Train: & Train: & Train: & Train: & Train: & Train: & Train: & Train: \\
& $200^{\circ}$ & $170^{\circ}$ & $150^{\circ}$ & $200^{\circ}$ & $170^{\circ}$ & $150^{\circ}$ & $200^{\circ}$ & $170^{\circ}$ & $150^{\circ}$ \\
\hline Whole mesh & 1.51 & $\mathbf{1 . 4 8}$ & 1.59 & 1.61 & $\mathbf{1 . 5 9}$ & 1.66 & 2.53 & 2.41 & $\mathbf{1 . 9 8}$ \\
Left atrium & 1.58 & $\mathbf{1 . 4 9}$ & 1.61 & 1.74 & $\mathbf{1 . 6 3}$ & 1.76 & 2.17 & 2.30 & $\mathbf{2 . 0 4}$ \\
Inf. Left PV & $\mathbf{1 . 8 7}$ & 1.88 & 2.00 & 1.99 & $\mathbf{1 . 8 3}$ & 1.88 & 2.65 & 2.81 & $\mathbf{2 . 2 0}$ \\
Inf. Right PV & 1.87 & $\mathbf{1 . 8 1}$ & 1.94 & 1.85 & 1.85 & $\mathbf{1 . 7 7}$ & 2.33 & 2.60 & $\mathbf{2 . 3 1}$ \\
Sup. Left PV & 1.41 & $\mathbf{1 . 3 8}$ & 1.65 & 1.65 & $\mathbf{1 . 4 1}$ & 1.70 & 2.11 & 2.13 & $\mathbf{2 . 0 8}$ \\
Sup. Right PV & 1.67 & $\mathbf{1 . 6 5}$ & 1.68 & 1.73 & $\mathbf{1 . 6 1}$ & 1.63 & 1.99 & 1.93 & $\mathbf{1 . 8 4}$ \\
Trachea & $\mathbf{1 . 3 5}$ & 1.36 & 1.46 & $\mathbf{1 . 3 8}$ & 1.51 & 1.52 & 3.00 & 2.55 & $\mathbf{1 . 8 6}$
\end{tabular}

These findings are also confirmed by visual inspection of the mesh outline as overlay on angiograms (Fig. 2) and slices of the reconstructed images (Fig. 3). The decreasing depth resolution in anterior-posterior direction can be observed in the transaxial slices for $\Delta=130^{\circ}, 110^{\circ}$ resulting in mesh deformations and larger segmentation errors. 

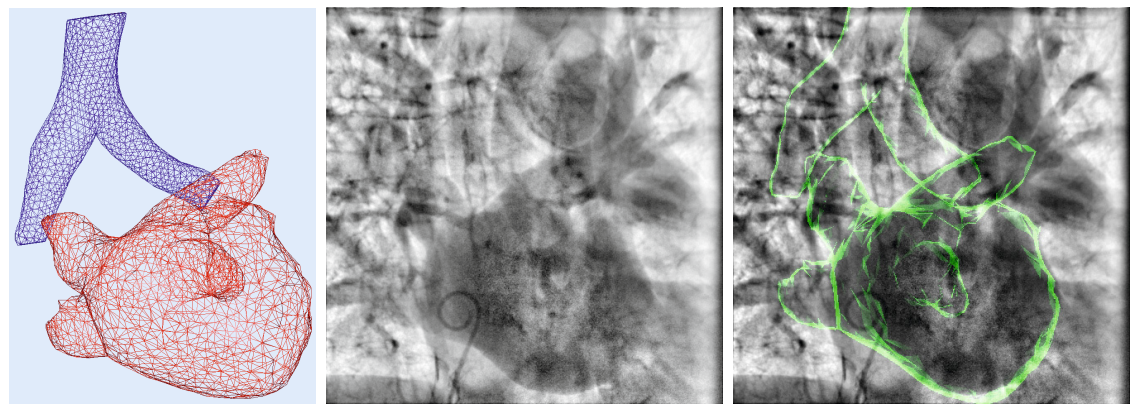

Fig. 2. Oblique view on the joint LAPV and trachea model as wire rendering (left), angiogram of the case shown in Fig. 3 post-processed by local contrast enhancement (middle), and overlay of corresponding adapted model trained on $200^{\circ}$ (right)

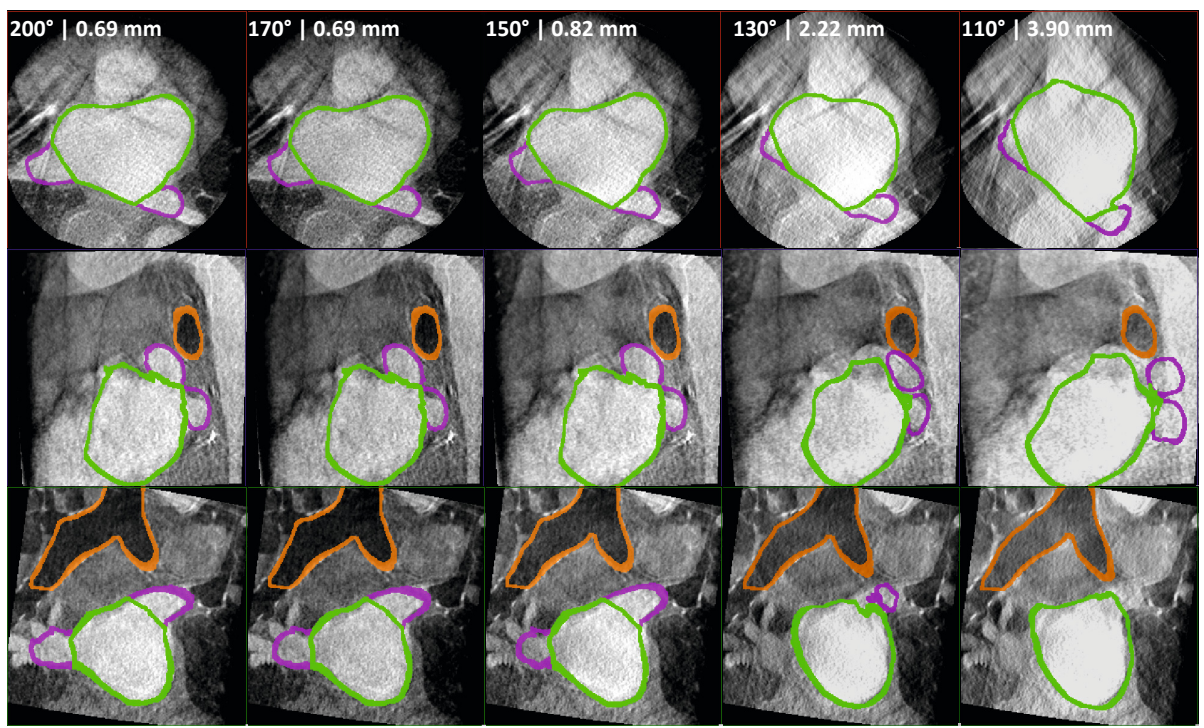

Fig. 3. Example overlay of segmentation results on the corresponding test data reconstructions using the trained model from $150^{\circ}$ with 10 -fold cross-correlation. Transaxial slices (top), sagittal (middle), coronal (bottom). In the top row the angular range $\Delta$ and the error $\epsilon_{\text {mean }}$ is given for the corresponding column.

\section{Summary and Conclusions}

A reduction of the angular range from $200^{\circ}$ to $170^{\circ}$ has only a minimal influence on the segmentation performance (from $1.5 \mathrm{~mm}$ to $1.6 \mathrm{~mm}$ ). If smaller angular ranges are used in the test images, the decrease in segmentation performance can be partly compensated by using a segmentation model trained in 
matched conditions. Thereby, for $150^{\circ}$ reconstructions, a segmentation error of about $2 \mathrm{~mm}$ is obtained. Hence, accepting this minor decrease in segmentation performance, the clinical workflow can be substantially simplified by avoiding large $\mathrm{C}$-arm angulations between $60^{\circ} \mathrm{RAO}$ and $100^{\circ} \mathrm{RAO}$.

\section{References}

1. Manzke, R., Meyer, C., Ecabert, O., Peters, J., Noordhoek, N.J., Thiagalingam, A., Reddy, V.Y., Chan, R.C., Weese, J.: Automatic Segmentation of Rotational X-Ray Images for Anatomic Intra-Procedural Surface Generation in Atrial Fibrillation Ablation Procedures. IEEE Trans. Med. Imag. 29(2), 260-272 (2010)

2. Delaney, A.H., Bresler, Y.: Globally Convergent Edge-Preserving Regularized Reconstruction: An Application to Limited-Angle Tomography. IEEE Trans. Imag. Proc. 7(2), 204-221 (1998)

3. Persson, M., Bone, D., Elmqvist, H.: Total variation norm for three-dimensional iterative reconstruction in limited view angle tomography. Phys. Med. Biol. 46, 853-866 (2001)

4. Snyder, D.L., O’Sullivan, J.A., Murphy, R.J., Politte, D.G., Whiting, B.R., Williamson, J.F.: Image reconstruction for transmission tomography when projection data are incomplete. Phys. Med. Biol. 51, 5603-5619 (2006)

5. Bachar, G., Siewerdsen, J.H., Daly, M.J., Jaffray, D.A., Irish, J.C.: Image quality and localization accuracy in C-arm tomosynthesis-guided head and neck surgery. Med. Phys. 34, 4664-4677 (2007)

6. Feldkamp, L., Davis, L., Kress, J.: Practical cone-beam algorithm. J. Opt. Soc. Am. A 1, 612-619 (1984)

7. Ecabert, O., Peters, J., Schramm, H., Lorenz, C., von Berg, J., Walker, M., Vembar, M., Olszewski, M., Subramanyan, K., Lavi, G., Weese, J.: Automatic model-based segmentation of the heart in CT images. IEEE Trans. Med. Imag. 27(9), 1189-1201 (2008)

8. Li, J.H., Haim, M., Movassaghi, B., Mendel, J.B., Chaudhry, G.M., Haffajee, C.I., Orlov, M.V.: Segmentation and registration of three-dimensional rotational angiogram on live fluoroscopy to guide atrial fibrillation ablation: A new online imaging tool. Heart Rhythm 6(2), 231-237 (2009)

9. Saalbach, A., Wächter-Stehle, I., Kneser, R., Mollus, S., Peters, J., Weese, J.: Optimizing GHT-Based Heart Localization in an Automatic Segmentation Chain. In: Fichtinger, G., Martel, A., Peters, T. (eds.) MICCAI 2011, Part III. LNCS, vol. 6893, pp. 463-470. Springer, Heidelberg (2011)

10. Peters, J., Ecabert, O., Meyer, C., Kneser, R., Weese, J.: Optimizing boundary detection via Simulated Search with applications to multi-modal heart segmentation. Medical Image Analysis 14(1), 70-84 (2010)

11. Meyer, C., Manzke, R., Peters, J., Ecabert, O., Kneser, R., Reddy, V.Y., Chan, R.C., Weese, J.: Automatic Intra-operative Generation of Geometric Left Atrium/Pulmonary Vein Models from Rotational X-Ray Angiography. In: Metaxas, D., Axel, L., Fichtinger, G., Székely, G. (eds.) MICCAI 2008, Part II. LNCS, vol. 5242, pp. 61-69. Springer, Heidelberg (2008)

12. Zheng, Y., Wang, T., John, M., Zhou, S.K., Boese, J., Comaniciu, D.: Multi-part Left Atrium Modeling and Segmentation in C-Arm CT Volumes for Atrial Fibrillation Ablation. In: Fichtinger, G., Martel, A., Peters, T. (eds.) MICCAI 2011, Part III. LNCS, vol. 6893, pp. 487-495. Springer, Heidelberg (2011) 